\title{
X-RAY EMISSION FROM AN EXPANDING SUPERGIANT SHELL IN IC 2574
}

\author{
Fabian Walter and JÜrgen KerP \\ Radioastronomisches Institut der Universität, Auf dem Hügel 71, D-53121 Bonn, Germany; \\ walter@astro.uni-bonn.de,jkerp@astro.uni-bonn.de \\ Neb DURIC \\ Department of Physics and Astronomy, University of New Mexico, Albuquerque, NM 87131; \\ duric@deneb.phys.unm.edu \\ ELIAS BRINKS \\ Departamento de Astronomía, Apartado Postal 144, Guanajuato, Gto. 36000, México; \\ National Radio Astronomy Observatory, Socorro, NM 87801; ebrinks@ astro.ugto.mx \\ AND \\ ULI KLEIN \\ Radioastronomisches Institut der Universität, Auf dem Hügel 71, D-53121 Bonn, Germany; \\ uklein@astro.uni-bonn.de \\ Received 1998 March 30; accepted 1998 June 5; published 1998 July 9
}

\begin{abstract}
We present a multiwavelength study of a supergiant shell within the violent interstellar medium of the nearby dwarf galaxy IC 2574, which is a member of the M81 group of galaxies. Neutral hydrogen (H I) observations obtained with the Very Large Array (VLA) reveal a prominent expanding supergiant H I shell in the northeast quadrant of IC 2574 which is thought to be produced by the combined effects of stellar winds and supernova explosions. It measures roughly $1000 \times 500 \mathrm{pc}$ in size and is expanding at $\sim 25 \mathrm{~km} \mathrm{~s}^{-1}$. The H I data suggest an age of $\sim 1.4 \times 10^{6} \mathrm{yr}$; the energy input must have been of order $(2.6 \pm 1) \times 10^{53}$ ergs. Massive star-forming regions, as traced by $\mathrm{H} \alpha$ emission, are situated predominantly on the rim of this $\mathrm{H}$ I shell. This supports the view that the accumulated $\mathrm{H}$ I on the rim has reached densities that are high enough for secondary star formation to commence. VLA radio continuum observations at $\lambda=6 \mathrm{~cm}$ show that these star-forming regions are the main sources of radio continuum emission in this galaxy. This emission is mainly thermal in origin. Soft X-ray emission from within the $\mathrm{H}$ i hole is detected by a pointed ROSAT PSPC observation. The emission is resolved, coinciding in size and orientation with the $\mathrm{H}$ I shell. These spatial properties suggest that the emission is generated by an X-ray-emitting plasma located within the $\mathrm{H}$ I shell, although a contribution from X-ray binaries cannot be completely ruled out. The X-ray luminosity within the $0.11-2.4 \mathrm{keV}$ energy range is $L_{\mathrm{X}}=(1.6 \pm 0.5) \times 10^{38}$ $\operatorname{ergs~} \mathrm{s}^{-1}$. The X-ray data are compatible with emission coming from a Raymond-Smith plasma at a temperature of about $\log (T[\mathrm{~K}])=6.8$ and a density of $n_{e} \sim 0.03 \mathrm{~cm}^{-3}$. The energy content of the coronal gas corresponds to $(4 \pm 2) \times 10^{53} \mathrm{ergs}$, or broadly in agreement with the energy input derived on the basis of the $\mathrm{H}$ I observations.

Subject headings: galaxies: individual (IC 2574) — galaxies: ISM — ISM: bubbles — ISM: structure — X-rays: ISM
\end{abstract}

\section{INTRODUCTION}

The interstellar medium (ISM) of galaxies is shaped by the aftermath of recent, massive star formation (SF). Huge cavities filled with coronal gas are believed to be blown in the ambient medium through strong stellar winds of the most massive stars as well as through subsequent Type II supernova (SN) explosions of stars more massive than $\approx 8 M_{\odot}$ (see Tenorio-Tagle $\&$ Bodenheimer 1988 for a review). The structures that form during such processes are often referred to as bubbles (diameter $\sim 10 \mathrm{pc}$ ), superbubbles $(\sim 200 \mathrm{pc})$, or supergiant shells $(\sim 1 \mathrm{kpc})$. A recent development is the finding that supergiant shells reach much larger sizes in dwarf galaxies than in more massive spiral galaxies (Puche et al. 1992; Walter \& Brinks 1998, hereafter WB98). This is attributed to dwarfs having a lower gravitational potential, which allows shells to grow more easily, and to the absence of differential rotation and spiral density waves, which prevents them from being destroyed prematurely. Dwarf galaxies are therefore ideally suited to study the origin and evolution of supergiant shells.

Cox \& Smith (1974) not only realized that the energy input of $\mathrm{SNe}$ creates cavities filled with hot gas but that the cooling time is relatively long $\left(10^{6}-10^{9} \mathrm{yr}\right.$, depending on density and temperature of the matter within), which allows the creation of a tunneling network (the Swiss cheese model). Weaver et al. (1977) discussed a model in which fast stellar winds are adiabatically shocked to temperatures of order $10^{6}-10^{7} \mathrm{~K}$. These winds then act like a piston, driving the expansion of the outer shell of swept-up ambient material. The basic picture still stands today, although several refinements have been proposed, e.g., by Chu \& Mac Low (1990), who calculate the expected X-ray emission from superbubbles.

The ISM that is swept up accumulates in the form of an expanding shell and forms the rim of a superbubble or supergiant shell. The resulting shocks on their rims may induce new generations of SF, or SF regions may be created simply via gravitational fragmentation (Elmegreen 1994; for an early discussion, see Mueller \& Arnett 1976).

Direct observational evidence for the theoretical scenarios mentioned above is still scarce. Very few, if any, supergiant shells have been detected to date through the emission of the coronal gas (see Bomans et al. 1996 for a review). In fact, it is the neutral and ionized gas that has proven to be the best tool for tracing the outlines of superbubbles. The best direct evidence has come from ROSAT PSPC observations, which 
give information about the plasma properties of the interior of some superbubbles. Examples are the supergiant shell LMC 4 (Bomans, Dennerl, \& Kürster 1994), the superbubbles N44 (Kim et al. 1998) and N11 (Mac Low et al. 1998), which are all three situated in the Large Magellanic Cloud (LMC), the supergiant shell SGS 2 in NGC 4449 (Bomans, Chu, \& Hopp 1997), and the possible supershell near Holmberg IX (Miller 1995). In the following, we will present the probable detection of coronal gas filling a supergiant shell within the nearby $(3.2$ Mpc) dwarf galaxy IC 2574.

\section{OBSERVATIONAL EVIDENCE FOR A HOT GAS-FILLED EXPANDING SUPERGIANT SHELL IN IC 2574}

\subsection{H I Observations}

The neutral interstellar medium of IC 2574 has been extensively studied by WB98 using H I observations obtained with the $\mathrm{NRAO}^{1}$ Very Large Array (VLA). An H I surface brightness map of IC 2574 is shown in Figure 1 (top left). WB98 find a total of $48 \mathrm{H}$ i holes in IC 2574, most of which are expanding. The most prominent of these holes (number 35 in their study) is the subject of this Letter. An H I map of the region around this hole is shown as a blowup in Figure 1 (middle left). The size of the hole is about $1000 \times 500$ pc (which corresponds to an effective radius $r_{\text {eff }} \approx 350 \mathrm{pc}$ ), and its radial expansion velocity is $\sim 25 \mathrm{~km} \mathrm{~s}^{-1}$. The dynamical age is therefore $\sim 1.4 \times 10^{7}$ yr. WB98 show that the $1 \sigma$ scale height of the $\mathrm{H}$ I disk is approximately $350 \mathrm{pc}$, or of the same order as the radius of the hole. Hence, the hole is still contained within the disk (no material lost due to blowout of the gas into the halo). Given the derived scale height and the ambient average $\mathrm{H} \mathrm{I}$ column density of $N_{\mathrm{H}} \approx 10^{21} \mathrm{~cm}$, the ambient $\mathrm{H}$ I volume density before the creation of the hole is estimated at $n_{\mathrm{H}_{\mathrm{I}}}=$ $0.5 \mathrm{~cm}^{-3}$. The $\mathrm{H}$ I mass that was present before the evacuation of the $\mathrm{H}$ I hole, which now must be largely accumulated on the rim, is therefore $\sim 2 \times 10^{6} M_{\odot}$. Using these numbers and correcting the $\mathrm{H}$ I mass for the contribution of primordial helium, we estimate the kinetic energy of the expanding shell to be $\approx 1.7 \times 10^{52}$ ergs.

No signature of an infalling high-velocity cloud is found in the $\mathrm{H}$ I data, and WB98 propose an internal origin for the creation of the hole. Using the numerical models of Chevalier (1974), who calculates the late phase of supernova remnants in which the shell accretes matter from both the interstellar medium and the hot interior, an energy input of $(2.6 \pm 1) \times$ $10^{53}$ ergs by a star-forming region is needed to create this hole. Note that the kinetic energy of the hole is of order $10 \%$ of this value, which is about half of the value originally predicted by Weaver et al. (1977). This energy is equivalent to $\sim 100$ Type II $\mathrm{SNe}$ and the strong stellar winds of their progenitors-a major SF event.

\section{2. $\mathrm{H} \alpha$ Observations}

In order to trace regions of current $\mathrm{SF}$ activity, $\mathrm{H} \alpha$ maps were obtained by WB98. A gray-scale representation of their $\mathrm{H} \alpha$ map is given in Figure 1 (top right). Although the global $\mathrm{SF}$ activity in IC 2574 is rather low, a ring of $\mathrm{H}$ II regions is prominent in the northeast quadrant of the galaxy. A blowup of the $\mathrm{H} \alpha$ emission around the same region as in the middle

\footnotetext{
${ }^{1}$ The National Radio Astronomy Observatory (NRAO) is operated by Associated Universities, Inc., under cooperative agreement with the National Science Foundation.
}

left-hand panel of Figure 1 is given in the middle right-hand panel. Note that virtually all $\mathrm{H} \alpha$ emission in that region is situated on the rim of the H I shell (as first noticed by Martimbeau, Carignan, \& Roy 1994). Three Wolf-Rayet stars have been detected in the most luminous $\mathrm{H}$ II region on the northern rim by Drissen, Roy, \& Moffat (1993). IUE spectra through the same region show the presence of $\mathrm{P}$ Cygni profiles, indicating the presence of strong stellar winds (Rosa, Joubert, \& Benvenuti 1984). Using the calibration by Miller \& Hodge (1994), the total $\mathrm{H} \alpha$ flux on the rim is $2.3 \times 10^{-12} \mathrm{ergs} \mathrm{cm}^{-2}$ $\mathrm{s}^{-1}$, which corresponds to a luminosity of $L(\mathrm{H} \alpha)=$ $7 \times 10^{5} L_{\odot}\left(L_{\odot}=3.85 \times 10^{33} \mathrm{ergs} \mathrm{s}^{-1}\right)$ or an energy equivalent of $\sim 50$ stars of spectral type O5 (WB98). This is almost one-third of the $\mathrm{H} \alpha$ luminosity of 30 Dor, which is believed to be the most active SF region in the Local Group (Chu \& Kennicutt 1994). The average volume densities derived on the rim of the shell are $3 \mathrm{~cm}^{-3}$ for the neutral $\mathrm{H} \mathrm{I}$ (southern part) and $2 \mathrm{~cm}^{-3}$ for the ionized hydrogen in the northern part. This suggests that most of the neutral gas component in the northern part of the hole has been ionized by the prominent SF regions.

\subsection{Radio Continuum Observations}

Radio continuum maps of IC 2574 were obtained at $\lambda=$ $6 \mathrm{~cm}$ using the VLA D array. A map of the $\lambda=6 \mathrm{~cm}$ emission is presented in Figure 1 (bottom right). We detect four radio sources in the region of the supergiant shell; their positions and flux densities (in parentheses) are B102459.7+684320 $(S=0.9 \quad$ mJy $), \quad$ B102503.1+684348 $\quad(S=1.1 \quad \mathrm{mJy})$, $\mathrm{B} 102507.5+684325(S=0.8 \mathrm{mJy})$, and B102507.7+684346 $(S=0.8 \mathrm{mJy})$. The flux densities are accurate to within $20 \%$. Three of the four sources coincide with regions of intense $\mathrm{H} \alpha$ emission on the rim of the shell. These sources have radio/ $\mathrm{H} \alpha$ flux ratios that are consistent with the radio emission being purely thermal (Caplan \& Deharveng 1986). This result confirms that the $\mathrm{H} \alpha$ emission traces photoionized gas and that these sources are indeed $\mathrm{H}$ II regions and not shock-ionized regions formed by the expansion of the hole.

The source furthest to the southwest, B102459.7+684320, is clearly nonthermal (see Fig. 1, bottom right), and its radio emission is well in excess of what one would expect from an $\mathrm{H}$ II region on the basis of the rather weak $\mathrm{H} \alpha$ emission. It most likely is a supernova remnant given its nonthermal nature, its appearance as a point source and a flux density that is consistent with the brighter SN remnants found in nearby galaxies (e.g., Duric et al. 1995; Lacey, Duric, \& Goss 1997).

\subsection{X-Ray Observations}

In order to examine the X-ray properties of IC 2574, we have analyzed a ROSAT PSPC observation toward IC 2574 (US600145P; total integration time $7.3 \mathrm{ks}$ ). The PSPC pointspread function at $\bar{E}=1.23 \mathrm{keV}$ is $\sim 28^{\prime \prime}$ (FWHM) across the area of interest. The pointed ROSAT PSPC observation reveals a significant X-ray feature that is coincident with the very center of the $\mathrm{H}$ i hole. A contour map of the X-ray emission is overlaid on the $\mathrm{H} \alpha$-emission in Figure 1, top right-hand panel (whole galaxy) and middle right-hand panel (blowup). An overlay with the $\mathrm{H} \mathrm{I}$ is presented in Figure 1 (bottom left). This X-ray feature has an angular extent of $\sim 45^{\prime \prime} \times 30^{\prime \prime}$ (FWHM), with its major axis aligned with the $\mathrm{H}$ I hole. Note that the emission is extended even if we account for the residual pointing uncertainties of the ROSAT telescope of $\sim 10^{\prime \prime}$ (G. Hasinger 1997, private communication). In addition, ROSAT's wobble direction during 

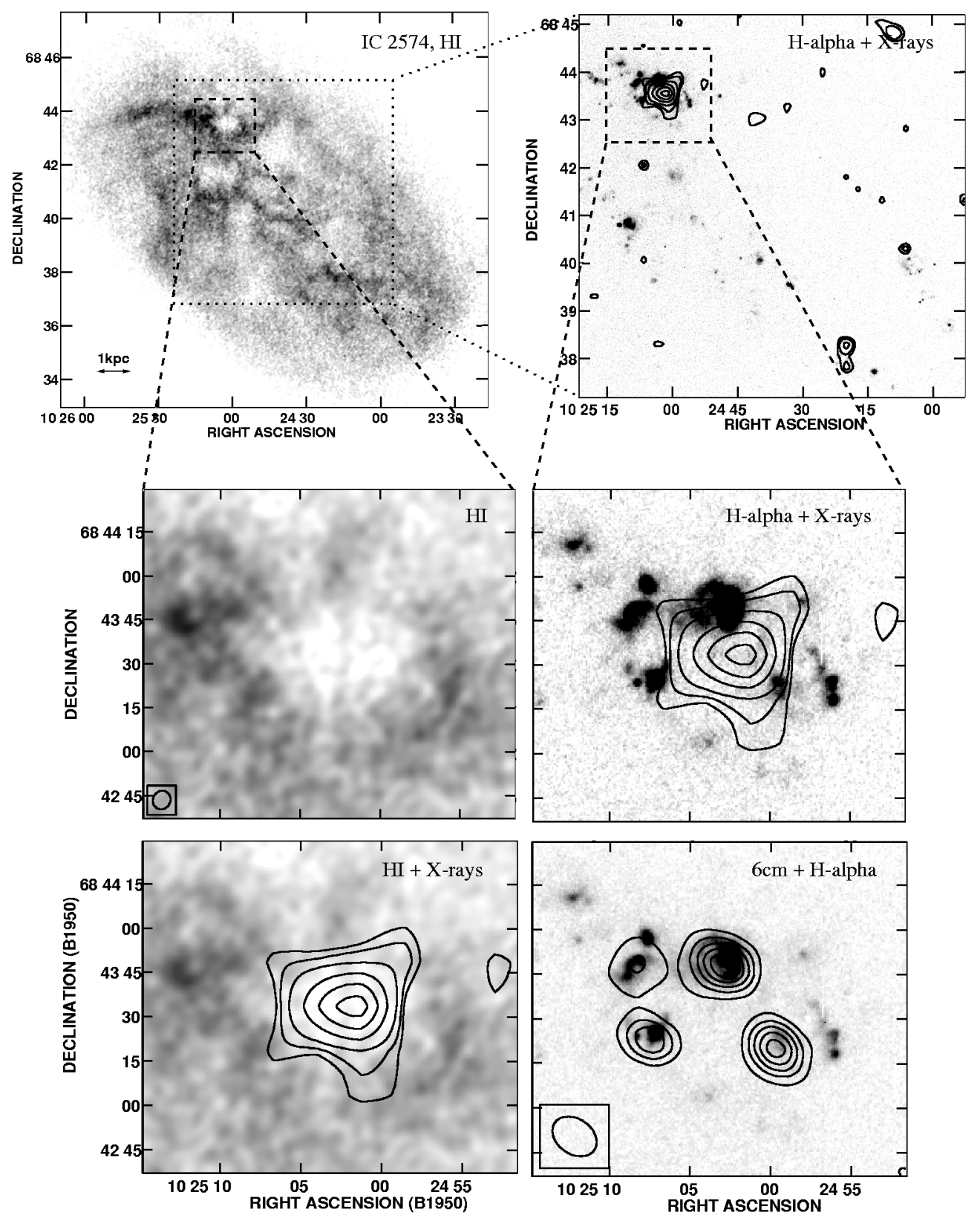

FIG. 1.-Top left: H I surface brightness map of IC 2574 as presented in WB98. Note that the ISM of IC 2574 is dominated by many hole-like structures. The dashed square indicates the $\mathrm{H}$ I hole that is the focus of this Letter. The dotted square shows the extent of the optical H $\alpha$ map. Top right: H $\alpha$ map of IC 2574 as presented in WB98 (gray scale). The contours (plotted at 15, 20, 30, 50, 70, 90\% of the peak emission) represent the X-ray emission of IC 2574 as observed with the ROSAT PSPC camera. The dashed square again indicates the supergiant shell that is the focus of this Letter. Middle left: Blowup of the $\mathrm{H}$ I surface brightness map around the prominent $\mathrm{H}$ I hole (the size of the beam is indicated in the lower left-hand corner). Middle right: Blowup of the H $\alpha$ image with $\mathrm{X}$ ray contours superposed. The area shown is identical to that in the middle left-hand panel; the X-ray contour levels are the same as in the top right-hand panel. Bottom left: X-ray contours overlaid on the $\mathrm{H}$ I hole. Bottom right: $\lambda=6 \mathrm{~cm}$ radio continuum emission of IC 2574 (contours) overlaid on the H $\alpha$ map (gray scale). The beam size is indicated in the lower left-hand corner. Contours are plotted at $0.14,0.22,0.31,0.39$ and $0.48 \mathrm{mJy}^{\mathrm{b}}$ beam ${ }^{-1}$.

the observations was perpendicular to the orientation of the major axis of the $\mathrm{H}$ I hole. Based on the ROSAT PSPC observations, we therefore find evidence for extended X-ray emission originating from the interior of the $\mathrm{H}$ I hole. However, higher resolution observations will be needed to confirm this.

IC 2574 is located at a high Galactic latitude $\left(b \approx+43^{\circ}\right)$, which means that the photoelectric absorption due to the Galactic interstellar medium is moderate. The Leiden/Dwingeloo H I $\lambda=21 \mathrm{~cm}$ line survey (Hartmann \& Burton 1997) gives an $\mathrm{H}$ I column density of $N_{\mathrm{H}}=2 \times 10^{20} \mathrm{~cm}^{-2}$ after separating the Galactic diffuse H I emission from that of IC 2574. Because of the apparently clear line of sight through the hole (see Fig. 1 , top left), we considered it justified to neglect any contribution to the H I column density within IC 2574.

To separate the source counts from that of the diffuse X-ray background, we performed an on/off analysis. This method also removes the contribution of the so-called non-cosmic X-ray background radiation (Snowden et al. 1994). In order to obtain quantitative information about the spectrum of the X-ray feature, we evaluated the standard ROSAT hardness ratios (HR1 
and HR2), which range from -1 (soft) to 1 (hard). We took this approach because the number of photons is too low for a standard analysis of the X-ray spectrum. We divided the ROSAT energy band into the three main energy bands, denoted as ROSAT C band $(0.25 \mathrm{keV}$, PI channels $11-41)$ the $\mathrm{M}$ band (0.75 keV, 52-90), and the $\mathrm{J}$ band (1.5 keV, 91-201). The evaluation of HR2, which is nearly unaffected by Galactic absorption, yields a value of HR2 $=(J-M) /(J+M)=$ $0.2 \pm 0.2$. This leads to a first estimate of the temperature of $\log (T[\mathrm{~K}]) \approx 6.8$. Adopting this estimate, we can correct the soft bands for photoelectric absorption by the Galactic ISM (Snowden et al. 1994). This analysis yields HR1 $=(J+$ $M-C) /(J+M+C)=0.2 \pm 0.4$ and the following photon counts for energies below and above $1 \mathrm{keV}$ : $I(E<1 \mathrm{keV}, 11-$ $90)=106 \pm 39$ and $I(E>1 \mathrm{keV}, 91-201)=38 \pm 9$ counts. This simple approach indicates that the X-ray feature emits most of its photons below $E=1 \mathrm{keV}$. Combined with the fact that the source is elongated, this suggests that a high-mass or even a low-mass X-ray binary is unlikely to be the source of the detected emission. It should be noted, though, that a possible contribution from X-ray binaries to the observed X-ray emission cannot be ruled out completely at this point.

Using the hardness ratios and assuming a reduced metallicity of $\sim 15 \%$ solar (as derived in a few $\mathrm{H}$ II regions in IC 2574 by Miller \& Hodge 1994), we calculate a plasma temperature range, according to Raymond \& Smith (1977), of $\log \left(T_{15 \%}[\mathrm{~K}]\right)=6.9-7.1$. If the X-ray plasma within the $\mathrm{H}$ I hole contains more metals, the temperature will be lower $\left[\log \left(T_{\text {solar }}[\mathrm{K}]\right)=6.4-6.8\right]$. Note that a higher abundance is more likely, since the interior of the shell has recently been enriched by the heavy elements ejected by the SNe that created the hole. As a result, the true temperature will lie somewhere in between; we adopt a temperature of $\log (T[\mathrm{~K}])=6.8 \pm$ 0.3 for the remainder of this Letter.

For a plasma of $\log (T[\mathrm{~K}])=6.8 \pm 0.3$ and a reduced metallicity, we evaluate an emission measure of $\mathrm{EM}=(0.65 \pm$ $0.15) \mathrm{cm}^{-6} \mathrm{pc}$. Assuming an extent of the line of sight through the X-ray cavity of $700 \mathrm{pc}$, we derive an electron density of $n_{e}=(0.03 \pm 0.01) \mathrm{cm}^{-3}$. This value is similar to the densities found in the supergiant shell SGS2 in NGC $4449\left(0.03 \mathrm{~cm}^{-3}\right)$ or in LMC $4\left(\approx 0.01 \mathrm{~cm}^{-3}\right)$ (Bomans et al. 1996). The total Xray luminosity of the interior of the hole is $L_{\mathrm{X}}(0.28-$ $2.4 \mathrm{keV})=(1.6 \pm 0.5) \times 10^{38} \mathrm{ergs} \mathrm{s}^{-1}$, which lies in between the values for SGS 2 in NGC $4449\left(\approx 7 \times 10^{38} \mathrm{ergs} \mathrm{s}^{-1}\right)$ and LMC $4\left(\approx 0.15 \times 10^{38} \mathrm{ergs} \mathrm{s}^{-1}\right)$. This luminosity is about 1 order of magnitude higher than predicted by theoretical models (see, e.g., the calculations by Chu et al. 1995), as is the case with most X-ray luminosities determined thus far (N11 being the only exception to date-see the discussion by Mac Low et al. 1998).

\section{DISCUSSION}

If we assume that the supergiant shell is the result of a recent SF event, the picture that emerges from the observations discussed above is the following: about $1.4 \times 10^{7} \mathrm{yr}$ ago (the dynamical age of the $\mathrm{H}$ I hole), a major SF event took place at the center of what today shows up as a prominent $\mathrm{H}$ I hole.
Since its creation, some $2.6 \times 10^{53}$ ergs of energy have been deposited by the most massive stars into the ambient ISM. About $10 \%$ of this energy is now still present in the form of the kinetic energy of the expanding shell. Note that the least massive stars that go off as $\mathrm{SNe}$ are most probably still present in the cavity, since their lifetime $\left(\approx 5 \times 10^{7} \mathrm{yr}\right)$ is somewhat longer then the dynamical age of the hole. In other words, there is still some energy input going on today that might explain why the derived temperature for the interior of the $\mathrm{H}$ i hole is at the upper end for supergiant shells. On the other hand, a larger than expected contribution from X-ray binaries may also explain the high-temperature estimate. Adopting a sound speed of $100 \mathrm{~km} \mathrm{~s}^{-1}$ for the coronal gas filling the hole, we derive a time of only $\approx 3 \times 10^{6} \mathrm{yr}$ before a sound wave actually reaches the rim of the shell. The gas has therefore had enough time to establish a relatively uniform distribution within the cavity.

For a plasma temperature of $\log (T[\mathrm{~K}])=6.8 \pm 0.3$ and an internal density of $(0.03 \pm 0.01) \mathrm{cm}^{-3}$, we derive an internal pressure of $P=2 n_{e} T \approx(4 \pm 2) \times 10^{5} \mathrm{~K} \mathrm{~cm}^{-3}$ (LMC 4: $P=2 \times 10^{4} \mathrm{~K} \mathrm{~cm}^{-3}$ ). This value has to be compared to the much lower pressure of the ambient warm ionized medium $\left(P \approx 10^{3}-10^{4} \mathrm{~K} \mathrm{~cm}^{-3}\right)$. This means that it is probably this hot gas that is still driving the expansion of the shell (see, e.g., Weaver et al. 1977).

The shell, as it is sweeping up material, has already reached the point at which secondary star formation can take place, as evidenced by the $\mathrm{H}$ II regions located along its periphery. The energy of the hot gas interior can be approximated by $3 / 2\left(2 n_{e}\right) k T V$ and yields a value of $(4 \pm 2) \times 10^{53}$ ergs. The model by Weaver et al. (1977) predicts that $\sim 50 \%$ of the total energy deposited by $\mathrm{SNe}$ is converted into thermal heating of the gas within the created superbubble. Our values indicate that, within the errors, most of the energy deposited by the SN explosions has indeed gone into the heating of the gas. Note that a small decrease in temperature and increase in the ambient density would bring these numbers in even closer agreement. In fact, it is the broad agreement of the observed numbers with those derived from theory that gives us added confidence that it is indeed hot diffuse gas that fills the cavity rather than a cluster of X-ray binaries. A definitive answer will have to await the better spectral and spatial resolution of future X-ray missions, such as the Advanced X-Ray Astrophysics Facility and $X$-Ray Multimirror Mission, which are expected to shed further light on this unique object.

F. W. gratefully acknowledges the Deutsche Forschungsgemeinschaft for the award of a stipendium in the Graduate School "The Magellanic Clouds and Other Dwarf Galaxies." E. B. acknowledges a grant from CONACyT (0460P-E) and thanks the Graduate School for financially supporting a visit to Bonn during which the foundations for this Letter were laid. We thank Dominik Bomans, You-Hua Chu, and MordecaiMark Mac Low for fruitful discussions during the Dwarf Galaxy workshop organized by the Graduate School and an anonymous referee for valuable comments that helped us to improve this Letter. 
Bomans, D. J., Chu, Y.-H., Magnier, E. A., \& Points, S. 1996, in Röntgenstrahlung from the Universe, ed. H. U. Zimmermann, J. E. Trümper, \& H. Yorke (Garching: MPI), 237

Caplan, J., \& Deharveng, L. 1986, A\&A, 155, 297

Chevalier, R. A. 1974, ApJ, 188, 501

Chu, Y.-H., Chang, H.-W., Su, Y.-L., \& Mac Low, M.-M. 1995, ApJ, 450, 156

Chu, Y.-H., \& Kennicutt, R. C. 1994, ApJ, 425, 720

Chu, Y.-H., \& Mac Low, M.-M. 1990, ApJ, 365, 510

Cox, D. P., \& Smith, B. W. 1974, ApJ, 189, L105

Drissen, L., Roy, J.-R., \& Moffat, A. F. J. 1993, AJ, 106, 1460

Duric, N., Gordon, M. S., Goss, W. M., Viallefond, F., \& Lacey, C. 1995, ApJ, 445, 173

Elmegreen, B. G. 1994, ApJ, 427, 384

Hartmann, D., \& Burton, W. B. 1997, An Atlas of Galactic Neutral Hydrogen (Cambridge: Cambridge Univ. Press)

Kim, S., Chu, Y.-H., Staveley-Smith, L., \& Smith, R. C. 1998, AJ, submitted Lacey, C., Duric, N., \& Goss, W. M. 1997, ApJS, 109, 417
Mac Low, M.-M., Chang, T. H., Chu, Y.-H., \& Points, S. D. 1998, ApJ, 493, 260

Martimbeau, N., Carignan, C., \& Roy, J.-R. 1994, AJ, 427, 656

Miller, B. W. 1995, ApJ, 446, L75

Miller, B. W., \& Hodge, P. 1994, ApJ, 427, 656

Mueller, M. W., \& Arnett, W. D. 1976, ApJ, 210, 670

Puche, D., Westpfahl, D., Brinks, E., \& Roy, J.-R. 1992, AJ, 103, 1841

Raymond, J. C., \& Smith, B. W. 1977, ApJS, 35, 419

Rosa, M., Joubert, M., \& Benvenuti, P. 1984, A\&AS, 57, 361

Snowden, S. L., McCammon, D., Burrows, D. N., \& Mendenhall, J. A. 1994, ApJ, 424, 714

Tenorio-Tagle, G., \& Bodenheimer, P. 1988, ARA\&A, 26, 145

Weaver, R., McCray, R., Castor, J., Shapiro, P., \& Moore, R. 1977, ApJ, 218, 377

Walter, F., \& Brinks, E. 1998, AJ, submitted (WB98) 